\title{
SOME PROPERTIES OF ASYMPTOTIC FUNCTIONS AND THEIR APPLICATIONS
}

\author{
LING-YAU CHAN
}

\begin{abstract}
In this paper we give complete characterizations, in terms of Dini numbers and integrals, of positive functions $\Phi(u)$ defined in $(0, \infty)$ satisfying the conditions: (i) $\Phi(u) / u^{a}$ is nondecreasing and (ii) $\Phi(u) / u^{b}$ is nonincreasing. By applying these results we obtain necessary and sufficient conditions for power series and trigonometric series to satisfy a certain Lipschitz condition, which include some known results of R. P. Boas, Jr. [1]. We also give complete characterizations of positive functions $\Phi(u)$ defined in $(-\infty, \infty)$ satisfying the conditions: (i) $\Phi(u) / e^{a u}$ is nondecreasing and (ii) $\Phi(u) / e^{b u}$ is nonincreasing.
\end{abstract}

1. Introduction. This paper is concerned with certain applications of properties of a special type of asymptotic functions. By $\Phi(u) \in Y[a, b]$ $(-\infty<a \leqslant b<\infty)$, we mean the positive function $\Phi(u)$ defined in $(0, \infty)$ satisfying: (i) $\Phi(u) / u^{a}$ is nondecreasing and (ii) $\Phi(u) / u^{b}$ is nonincreasing. By $\Phi(u) \in Y\langle a, b\rangle(-\infty<a<b<\infty)$, we mean that $\Phi(u) \in Y[a+\varepsilon, b-\varepsilon]$ for some $\varepsilon>0$. (In some cases $\Phi(u)$ is defined only for all large $u$ but not in the whole range $(0, \infty)$. If this happens and ambiguity may arise, we shall specify and write: $\Phi(u) \in Y[a, b]$ (for sufficiently large $u$ ), etc.) The above classes of asymptotic functions have various applications in analysis and differential equations [4]-[6], [9]-[11], [2, \$8], and are closely related to other classes of functions, viz., the class of $R-0$ varying functions [12, p. 92], the asymptotic classes $M(a, b)$ and $Z(a, b)$ introduced by J. Marcinkiewicz [13, II, p. 116] and S. Koizumi [8, pp. 194-195]. The main object of this paper is to study functions in the class $Y[a, b]$.

We say that $\phi(u) \in R-0(R-0$ varying at infinity) if $\phi(u)$ is positive and measurable on $[A, \infty)$ for some $A>0$, and $m \leqslant \phi(\lambda u) / \phi(u)<M$ for all $\lambda$ such that $1 \leqslant \lambda \leqslant c$, where $1<c<\infty, 0<m<1<M<\infty$. We say that $\phi(u) \in M(a, b)(0 \leqslant a<b<\infty)$ if $\phi(u)$ is continuous, nondecreasing and not identically zero in $[0, \infty)$ such that $\phi(0)=0$, and $\int_{u}^{\infty} t^{-b-1} \phi(t) d t=$ $O\left(u^{-b} \phi(u)\right), \int_{1}^{u} t^{-a-1} \phi(t) d t=O\left(u^{-a} \phi(u)\right)$ as $u \rightarrow \infty$. By $Z(a, b)$ we denote the subclass of $M(a, b)$, in which $\phi(u)>0$ when $u>0$, and $\int_{u}^{1} t^{-b-1} \phi(t) d t$ $=O\left(u^{-b} \phi(u)\right), \int_{0}^{u} t^{-a-1} \phi(t) d t=O\left(u^{-a} \phi(u)\right)$ as $u \rightarrow 0^{+}$. It has been proved [3, Remark 3] that if $\phi(u) \in M(a, b)$ then $\phi(2 u)=O(\phi(u))$ as $u \rightarrow \infty$, and if

\footnotetext{
Received by the editors September 26, 1977.

AMS (MOS) subject classifications (1970). Primary 26A12, 42A32; Secondary 26A84, 26A86, $42 \mathrm{~A} 16$.

Key words and phrases. Asymptotic functions, interpolation, trigonometric series, power series, Lipschitz condition.
} 
$\phi(u) \in Z(a, b)$ then in addition $\phi(2 u)=O(\phi(u))$ as $u \rightarrow 0^{+}$.

It is proved (Theorem 4(a) below in the Appendix) that the classes $Y[a, b]$, $M(a, b)$ (and a fortiori the class $Z(a, b))$ are subclasses of the class $R-0$. In [12, Theorem A.2, pp. 94-95] one finds another class of functions more general than $Y[a, b]$ and still contained in $R-0$. On the other hand, Theorem 4(b) in the Appendix shows that the class $R-0$ is covered by the $Y$ class in the following sense: Given any $\phi(u) \in R-0$, we can find constants $a, b$, positive constants $K_{1}, K_{2}$, and a function $\Phi(u) \in Y[a, b]$ such that the relation $K_{1} \Phi(u) \leqslant \phi(u) \leqslant K_{2} \Phi(u)$ is satisfied for all large $u$. In view of this, we can say that the class $Y$ and the class $R-0$ are equivalent.

Recently, it has also been found [3, Theorem 2, Lemma 1] that the class $Y\langle a, b\rangle(0 \leqslant a<b<\infty)$ is equivalent to the classes $M(a, b)$ and $Z(a, b)$. More precisely, this is:

(i) $Y\langle a, b\rangle \subset Z(a, b) \subset M(a, b)$;

(ii) given any $\phi(u) \in Z(a, b)$ or $\phi(u) \in M(a, b)$, there exists $\Phi(u) \in$ $Y\langle a, b\rangle$ and positive constants $K_{3}, K_{4}$, such that $K_{3} \Phi(u) \leqslant \phi(u) \leqslant K_{4} \Phi(u)$ for $u \geqslant 0$ or for sufficiently large $u$ according as $\phi(u) \in Z(a, b)$ or $\phi(u) \in$ $M(a, b)$.

Hence functions in the classes $Y\langle a, b\rangle$ and $Z(a, b)$ (or $M(a, b)$ ) can be interchanged in many cases.

The main advantage of the classes $M$ and $Z$ is in the powerful interpolation theorems due to J. Marcinkiewicz [13, II, p. 116] and S. Koizumi [8, Theorem 4]. In spite of this, results which follow from these interpolation theorems are usually weaker than those that follow from properties of $Y$ class. For example, by the use of $Y$ class Mulholland proved [9, Lemma 2] the classical Hardy's inequality

$$
\int_{0}^{a} x^{-c} \Phi(F(x)) d x \leqslant K \int_{0}^{a} x^{-c} \Phi(x f(x)) d x \text { for } \Phi(u) \in Y[1, k],
$$

whereas by application of Koizumi's interpolation theorem we can only prove this inequality for $\Phi(u) \in Z(1, k)$ and for $\Phi(u) \in Y\langle 1, k\rangle$. Moreover, in the case when no suitable quasi-linear operator can be defined so that these interpolation theorems are not applicable, we have to make use of properties of $Y$ class instead of properties of $Z$ and $M$ classes. Owing to these reasons, the study of properties of $Y$ class is found to be useful.

In this paper we shall prove two necessary and sufficient conditions for functions to belong to the class $Y[a, b]$. As exampling applications of these results of R. P. Boas, Jr. (cf. Remarks 2, 3, 4 below). These results do not seem to follow from the above mentioned interpolation theorems. We also give necessary and sufficient conditions for functions to belong to $E[a, b]$ $(-\infty<a \leqslant b<\infty)$, where $E[a, b]$ denotes the class of all positive functions $\Phi(u)$ defined in $(-\infty, \infty)$ satisfying the conditions: (i) $\Phi(u) / e^{a u}$ is nondecreasing and (ii) $\Phi(u) / e^{b u}$ is nonincreasing. Such functions were considered, for example, in [11]. 
Throughout this paper we use $D_{i} F(u)(i=1,2,3,4)$ to denote the four Dini numbers $D^{+} F(u), D_{+} F(u), D^{-} F(u), D_{-} F(u)$ of any function $F(u)$.

ACKNOWLEDGEMENT. The author wishes to express his thanks to the referee for pointing out and supplying the proof of Theorem 4(a). The referee has also pointed out to the author that there exists a class of functions more general than $Y[a, b]$ and still contained in $R-0$.

The author wishes to express his gratitude to Professor Y. M. Chen and Dr. M. C. Liu for their valuable suggestions in the presentation of this paper.

\section{Main results.}

TheOREM 1. Suppose that $-\infty<a \leqslant b<\infty$ and that $\Phi(u)$ is a positive function defined in $(0, \infty)$. Then $\Phi(u) \in Y[a, b]$ if and only if

$$
a \Phi(u) / u \leqslant D_{i} \Phi(u) \leqslant b \Phi(u) / u \quad(i=1,2,3,4)
$$

for all $u \in(0, \infty)$.

THEOREM 2. Under the hypotheses of Theorem $1, \Phi(u) \in Y[a, b]$ if and only if

$$
\Phi(u) \in L(\alpha, \beta)
$$

and

$$
(a+1) \int_{\alpha}^{\beta} \Phi(t) d t \leqslant \beta \Phi(\beta)-\alpha \Phi(\alpha) \leqslant(b+1) \int_{\alpha}^{\beta} \Phi(t) d t
$$

for all $\alpha \in(0, \infty), \beta \in(0, \infty), \alpha<\beta$.

DeFinition (GENERALIZED LiPSCHITZ CONDITION, CF. [4, p. 401]). Let $\theta(u)$ $\in Y[a, b]$, where $0 \leqslant a \leqslant b \leqslant 1$. Suppose that $f(x)$ is a function defined in some set $E \subset(-\infty, \infty)$. By $f(x) \in \operatorname{Lip} \theta$ we mean that for every small $\delta>0, \omega(\delta ; f)=\sup |f(x)-f(y)| \leqslant A \theta(\delta)$, where $A>0$ depends on $E$ and $\theta(u)$ only, and the supremum is taken for all $x \in E, y \in E$ and $|x-y| \leqslant \delta$.

TheOREM 3. Let $0<\gamma_{1} \leqslant \gamma_{2}<1$ and $\theta(u) \in Y\left[\gamma_{1}, \gamma_{2}\right]$. Suppose that $c_{n} \geqslant$ $0(n=0,1,2, \ldots), h(x)=\sum_{n=0}^{\infty} c_{n} x^{n}$ converges in $[0,1]$, and $f(x)=\sum_{n=0}^{\infty} c_{n}$ $\cos n x, g(x)=\sum_{n=0}^{\infty} c_{n} \sin n x$. Then the following statements are equivalent:

$$
\begin{aligned}
\sum_{k=n}^{\infty} c_{k}=O(\theta(1 / n)) & (n=1,2, \ldots) ; \\
\sum_{k=1}^{n} k c_{k}=O(n \theta(1 / n)) & (n=1,2, \ldots) ; \\
h(x) & \in \operatorname{Lip} \theta \quad(x \in[0,1]) ; \\
f(x) & \in \operatorname{Lip} \theta \quad(x \in[0, \pi]) ; \\
g(x) & \in \operatorname{Lip} \theta \quad(x \in[0, \pi]) .
\end{aligned}
$$

Hence, either (2.7) or (2.8) is equivalent to 


$$
\sum_{n=0}^{\infty} c_{n} e^{i n x} \in \operatorname{Lip} \theta \quad(x \in[0, \pi]) .
$$

REMARK 1. In Theorem 3, under the hypothesis that $\Sigma_{0}^{\infty} c_{n} x^{n}$ converges in $[0,1]$ we have $\Sigma_{0}^{\infty} c_{n}<\infty$. This implies [13, I, p. 326] that both $\Sigma_{0}^{\infty} c_{n} \cos n x$ and $\sum_{0}^{\infty} c_{n} \sin n x$ are Fourier series of $f(x)$ and $g(x)$ respectively. In fact, Theorem 3 is meaningful only when $\Sigma_{0}^{\infty} c_{n} x^{n}$ converges in $[0,1]$. For if $\sum_{0}^{\infty} c_{n}=\infty$ then (2.4), (2.6), (2.7) cannot be satisfied, and by the equivalence, (2.5), (2.8), (2.9) cannot be satisfied also.

REMARK 2. If we put $\theta(u)=u^{\gamma}(0<\gamma<1)$ then the trigonometric series part of Theorem 3 reduces to Theorem 1 in [1]. Lipschitz condition for the power series $\Sigma_{0}^{\infty} c_{n} x^{n}$ was not considered in [1]. It is interesting to compare the form of Theorem 3 with that of the theorems stated in [7].

THEOREM 4. Suppose that $-\infty<a \leqslant b<\infty$ and that $\Phi(u)$ is a positive function defined in $(-\infty, \infty)$. Then $\Phi(u) \in E[a, b]$ if and only if

$$
a \Phi(u) \leqslant D_{i} \Phi(u) \leqslant b \Phi(u) \quad(i=1,2,3,4)
$$

for all $u \in(-\infty, \infty)$.

THEOREM 5. Under the hypotheses of Theorem 4, $\Phi(u) \in E[a, b]$ if and only if

$$
\Phi(u) \in L(\alpha, \beta)
$$

and

$$
a \int_{\alpha}^{\beta} \Phi(t) d t \leqslant \Phi(\beta)-\Phi(\alpha) \leqslant b \int_{\alpha}^{\beta} \Phi(t) d t
$$

for all $\alpha \in(-\infty, \infty), \beta \in(-\infty, \infty), \alpha<\beta$.

3. Proofs of theorems. It is known [3, Theorem 1] that if $\Phi(u) \in Y[a, b]$ $(-\infty<a \leqslant b<\infty)$, then $\Phi(u)$ is absolutely continuous in any closed interval which does not contain the origin. This property will be used in the proofs of Theorems 1 and 2.

Proof of Theorem 1. First let $\Phi(u) \in Y[a, b]$. Since $\Phi(u) / u^{a}$ is nondecreasing in $(0, \infty)$, for any arbitrary fixed $u \in(0, \infty)$ and any small increment $\Delta$ (positive or negative) we have $\left\{\Phi(u+\Delta)(u+\Delta)^{-a}-\right.$ $\left.\Phi(u) u^{-a}\right\} / \Delta \geqslant 0$. Expanding $(1+\Delta / u)^{-a}$ we obtain

$$
\begin{aligned}
& \left\{\Phi(u+\Delta)(1+\Delta / u)^{-a}-\Phi(u)\right\} \Delta^{-1} u^{-a} \\
& \quad=\left\{[\Phi(u+\Delta)-\Phi(u)] / \Delta-a u^{-1} \Phi(u+\Delta)+O\left(\Delta / u^{2}\right) \Phi(u+\Delta)\right\} u_{1}^{-a} \\
& \quad \geqslant 0 \text { for all small } \Delta .
\end{aligned}
$$

Thus, letting $\Delta \rightarrow 0$ and taking into account the continuity of $\Phi(u)$ we have $D_{i} \Phi(u)-a \Phi(u) / u \geqslant 0(i=1,2,3,4)$. Similarly, for any $u \in(0, \infty)$ we have $D_{i} \Phi(u)-b \Phi(u) / u \leqslant 0(i=1,2,3,4)$. Hence (2.1) follows.

For the converse, assuming that $g(u)=\Phi(u) / u^{a}$ is not nondecreasing in 
$(0, \infty)$, i.e., there exists $\alpha \in(0, \infty), \beta \in(0, \infty), \alpha<\beta$, such that $g(\alpha)>$ $g(\beta)$. Therefore there exists $i_{0} \in\{1,2,3,4\}$ and $\xi \in[\alpha, \beta] \subset(0, \infty)$ such that $D_{i_{0}} g(\xi) \leqslant-\varepsilon<0$, where $\varepsilon=(g(\alpha)-g(\beta)) /(\beta-\alpha)>0$.

Hence there exists a sequence $\left\{\Delta_{j}\right\}^{\infty}$ of small increments (positive or negative) tending to zero, such that the following holds:

$$
\begin{aligned}
\{g(\xi & \left.\left.+\Delta_{j}\right)-g(\xi)\right\} / \Delta_{j} \\
& =\left\{\Phi\left(\xi+\Delta_{j}\right)\left(1+\Delta_{j} / \xi\right)^{-a}-\Phi(\xi)\right\}\left(\Delta_{j} \xi^{a}\right)^{-1} \\
& =\left\{\left[\Phi\left(\xi+\Delta_{j}\right)-\Phi(\xi)\right] / \Delta_{j}-a \Phi\left(\xi+\Delta_{j}\right) / \xi+O\left(\Delta_{j} / \xi^{2}\right)\right\} \xi^{-a} \\
& \leqslant-\frac{1}{2} \varepsilon \text { for all } \Delta_{j} .
\end{aligned}
$$

It follows that

$$
\begin{aligned}
& \left\{\left[\Phi\left(\xi+\Delta_{j}\right)-\Phi(\xi)\right] / \Delta_{j}-a \Phi\left(\xi+\Delta_{j}\right) / \xi\right\} \\
& \leqslant-\frac{1}{2} \varepsilon \xi^{a}+O\left(\Delta_{j}\right) \leqslant-\frac{1}{4} \varepsilon \xi^{a}<0 \text { for all } \Delta_{j} .
\end{aligned}
$$

Thus (2.1) cannot be satisfied for $i_{0} \in\{1,2,3,4\}$ and $\xi \in(0, \infty)$. By similar argument we can show that if $\Phi(u) / u^{b}$ is not nonincreasing then (2.1) cannot be satisfied for some $i \in\{1,2,3,4\}$ and for some $u \in(0, \infty)$.

This completes the proof of Theorem 1 .

Proof of Theorem 2. We first let $\Phi(u) \in Y[a, b]$. Let $\alpha$ be an arbitrary fixed point in $(0, \infty)$. Since $\Phi(u)$ is continuous in $[\alpha, \infty), \Phi(u) \in L(\alpha, \beta)$ for any $\beta \in(\alpha, \infty)$. Let $C$ be a real constant, and define a function $F(u)$ by

$$
F(u)=u \Phi(u)-C \int_{\alpha}^{u} \Phi(t) d t-\alpha \Phi(\alpha) \text { for } u>\alpha .
$$

As $D_{i} F(u)=\Phi(u)+u D_{i} \Phi(u)-C \Phi(u)$, by Theorem 1 we see that $D_{i} F(u) \geqslant$ 0 if $C=a+1$ and $D_{i} F(u) \leqslant 0$ if $C=b+1(i=1,2,3,4)$. Therefore $F(u)$ is nondecreasing when $C=a+1$ and is nonincreasing when $C=b+1$. Since $F(\alpha)=0,(2.3)$ follows immediately.

Next, assume that both (2.2) and (2.3) hold. For any arbitrary fixed $\alpha$, $\beta \in(0, \infty), \alpha \leqslant v<w \leqslant \beta,(2.3)$ gives

$$
\begin{aligned}
& w(\Phi(w)-\Phi(v)) \leqslant|b+1| \int_{v}^{w} \Phi(t) d t-\Phi(v)(w-v) \text { and } \\
& w(\Phi(w)-\Phi(v)) \geqslant-|a+1| \int_{v}^{w} \Phi(t) d t-\Phi(v)(w-v) .
\end{aligned}
$$

Furthermore, $\Phi(u)$ must be bounded in $[\alpha, \beta]$, for otherwise it would follow from (2.3) that $\int_{\alpha}^{\beta} \Phi(t) d t=\infty$. Thus it follows from (3.1) that $\Phi(u)$ is absolutely continuous in $[\alpha, \beta]$, and therefore $\int_{\alpha}^{\beta} \Phi(t) d t /(\beta-\alpha) \rightarrow \Phi(u)$ when $\alpha \rightarrow u^{-}, \beta \rightarrow u^{+}$, where $u \in(\alpha, \beta)$. (2.3) gives 


$$
\begin{aligned}
(a+1) \int_{\alpha}^{\beta} \Phi(t) d t /(\beta-\alpha)- & \Phi(\alpha) \leqslant \beta[\Phi(\beta)-\Phi(\alpha)] /(\beta-\alpha) \\
& \leqslant(b+1) \int_{\alpha}^{\beta} \Phi(t) d t /(\beta-\alpha)-\Phi(\alpha) .
\end{aligned}
$$

By the absolute continuity of $\Phi(u)$ and by taking the limits in (3.2) when $\alpha \rightarrow u^{-}, \beta \rightarrow u^{+}(u \in(\alpha, \beta))$, we obtain

$$
(a+1) \Phi(u)-\Phi(u) \leqslant u D_{i} \Phi(u) \leqslant(b+1) \Phi(u)-\Phi(u)
$$

for $i=1,2,3,4$. Then, it follows from Theorem 1 that $\Phi(u) \in Y[a, b]$.

This completes the proof of Theorem 2.

The proofs of Theorem 4 and Theorem 5 run in similar ways, so we omit the details.

The following Lemmas 1 and 2 are required in the proof of Theorem 3.

LEMMA 1. Let $0<\beta_{1} \leqslant \beta_{2}<\delta_{1} \leqslant \delta_{2}<\infty, \phi(u) \in Y\left[\beta_{1}, \beta_{2}\right], \psi(u) \in$ $Y\left[\delta_{1}, \delta_{2}\right]$, and let $c_{k} \geqslant 0(k=1,2, \ldots)$. Then

$$
\sum_{k=n}^{\infty} c_{k}=O(\phi(n) / \psi(n)) \quad(n=1,2, \ldots)
$$

is equivalent to

$$
\sum_{k=1}^{n} \psi(k) c_{k}=O(\phi(n)) \quad(n=1,2, \ldots)
$$

REMARK 3. If we put $\phi(u)=u^{\beta}, \psi(u)=u^{\delta}(0<\beta<\delta<\infty)$, then Lemma 1 here reduces to Lemma 1 in [1]. Lemma 1 here can be proved by the technique of partial summation (for a standard argument see [1, pp. 476-477]). In doing so it is required to estimate the quantities $\sum_{k=n}^{N} \phi(k)(k \psi(k$ $+1))^{-1}$ and $\sum_{k=2}^{n} \phi(k) / k$, which are in fact by Theorem 2 not exceeding $A(\phi(n) / \psi(n)-\phi(N) / \psi(N))$ and $A^{\prime} \phi(1)+A^{\prime \prime}(\phi(n)-\phi(1))$ respectively, where $A, A^{\prime}, A^{\prime \prime}$ are positive numbers independent of $n$ and $N$. As all the arguments are standard we omit the details.

Lemma 2. Let $0<\beta_{1} \leqslant \beta_{2}<2, \eta(u) \in Y\left[\beta_{1}, \beta_{2}\right], c_{n} \geqslant 0(n=1,2, \ldots)$, and $\sum_{1}^{\infty} c_{n}<\infty$. Then

$$
\sum_{n=1}^{\infty} c_{n}(1-\cos n x)=O(\eta(x)) \quad(x \in[0, \pi])
$$

is equivalent to

$$
\sum_{k=n}^{\infty} c_{k}=O(\eta(1 / n)) \quad(n=1,2, \ldots)
$$

RemarK 4. If we put $\eta(u)=u^{\beta}(0<\beta<2)$ then Lemma 2 here reduces to Lemma 2 in [1]. Making use of Lemma 1 (with $\psi(u)=u^{2}, \phi(u)=u^{2} \eta(1 / u)$ ), Lemma 2 here can be proved by following the arguments in [1, p. 468], so we omit the details. 
Proof of TheOrem 3. First of all, we see that the equivalence of (2.4) and (2.5) is merely a special case of Lemma 1 in which $\phi(u)=u \theta(1 / u)$ and $\psi(u)=u$.

To show that (2.6) implies (2.5), we put $x_{1}=1-2 /(n+2), x_{2}=1-1 /(n$ $+2)(n=1,2, \ldots)$. Since $h(x) \in \operatorname{Lip} \theta$, we have

$$
\begin{aligned}
A \theta(1 /(n+2)) & \geqslant h\left(x_{2}\right)-h\left(x_{1}\right) \\
& =\sum_{k=0}^{\infty}\left[(1-1 /(n+2))^{k}-(1-2 /(n+2))^{k}\right] c_{k} \\
& \geqslant \sum_{k=1}^{\infty}(n+2)^{-1} k(1-2 /(n+2))^{k-1} c_{k} \\
& \geqslant(n+2)^{-1} \sum_{k=1}^{n} k(1-2 /(n+2))^{n+2} c_{k} \\
& \geqslant A^{\prime} n^{-1} \sum_{k=1}^{n} k c_{k} \quad(n=1,2, \ldots),
\end{aligned}
$$

where $A>0, A^{\prime}>0$ are independent of $n$. Hence (2.5) follows.

Next, we shall show that (2.4) and (2.5) together imply (2.6). We let $x_{1} \in[0,1], x_{2}=x_{1} \pm t \in[0,1]$, where without loss of generality we put $t=1 / n(n=1,2, \ldots)$. We have

$$
\begin{aligned}
\left|h\left(x_{2}\right)-h\left(x_{1}\right)\right| & =\sum_{k=1}^{\infty}\left|\left(x_{1} \pm t\right)^{k}-x_{1}^{k}\right| c_{k} \\
& \leqslant \sum_{k=1}^{n}\left|\left(x_{1} \pm t\right)^{k}-x_{1}^{k}\right| c_{k}+\sum_{k=n}^{\infty} c_{k} \\
& \leqslant \sum_{k=1}^{n} k t\left(x_{1}+t\right)^{k-1} c_{k}+O(\theta(1 / n)) \\
& \leqslant n^{-1} O(n \theta(1 / n))+O(\theta(1 / n))=O\left(\theta\left(\left|x_{2}-x_{1}\right|\right)\right) .
\end{aligned}
$$

Hence $h(x) \in \operatorname{Lip} \theta$.

The proof of the remaining part of Theorem 3 concerning trigonometric series follows the same line as the proof of Theorem 1 in [1] (but Lemma 2 in the present paper is applied instead of Lemma 2 in [1]), so we omit the details.

Appendix. In this Appendix we shall prove the following theorem:

THEOREM 4. (a) (i) Let $-\infty<a \leqslant b<\infty$. Then we have $Y[a, b] \subset R-0$. (ii) Let $0 \leqslant a<b<\infty$. Then we have $M(a, b) \subset R-0$.

(b) For any $\phi(u) \subset R-0$, there exist constants $a, b$, positive constants $K_{1}$, $K_{2}$, and a continuously differentiable function $\Phi(u) \in Y[a, b]$, such that the relation $K_{1} \phi(u) \leqslant \Phi(u) \leqslant K_{2} \phi(u)$ is satisfied for all large $u$.

Proof. (a) (i) Let $\Phi(u) \in Y[a, b]$, and $1 \leqslant \lambda<c$. Since $\Phi(u) / u^{a}$ is nondecreasing, $\Phi(\lambda u) /(\lambda u)^{a} \geqslant \Phi(u) / u^{a}$, and hence $\Phi(\lambda u) / \Phi(u)>\lambda^{a}>c^{-|a|}$ 
$=m$. In the same way, since $\Phi(u) / u^{b}$ is nonincreasing, $\Phi(\lambda u) / \Phi(u)<c^{|b|}=$ $M$. Thus $\Phi(u) \in R-0$.

(ii) Let $\phi(u) \in M(a, b)$, and $1 \leqslant \lambda \leqslant 2$. Then since $\phi(2 u)=O(\phi(u))$ as $u \rightarrow \infty$, we have $1 \leqslant \phi(\lambda u) / \phi(u) \leqslant \phi(2 u) / \phi(u) \leqslant M$ when $u$ is sufficiently large. So, $\phi(u) \in R-0$.

(b) Suppose that $\phi(u)(>0)$ satisfies

$$
m \phi(u) \leqslant \phi(\lambda u) \leqslant M \phi(u)
$$

for all $\lambda \in[1, c]$ and $u \geqslant A$, where $c, m, M, A$ are positive constants and $c>1,0<m<M<\infty$ (in this proof we do not require $m<1$ and $1<$ $M)$.

Since $\phi(u) \in R-0$, it is integrable over any finite subinterval of $[A, \infty)$ $[12$, p. 93]. Define

$$
\Phi(u)=\int_{u}^{c u} \int_{v}^{c v} \phi(t) t^{-2} d t d v \quad(u \geqslant A) .
$$

We shall show that this function satisfies our requirements.

Since $\Phi(u)$ is a double integral, it has a continuous derivative.

It is not difficult to deduce from (A.1) that

$$
\begin{aligned}
K_{1} \phi(u) & =m^{2}\left(1-c^{-1}\right) \log c \phi(u) \\
& \leqslant \Phi(u) \leqslant M^{2}\left(1-c^{-1}\right) \log c \phi(u)=K_{2} \phi(u) .
\end{aligned}
$$

Let $\alpha$ be any (fixed) real number. Straightforward calculation shows that

$$
D=\frac{d}{d u}\left(\Phi(u) / u^{\alpha}\right)=\{[\cdots] u-\alpha \Phi(u)\} / u^{\alpha+1},
$$

where $[\cdots]=\left[c \int_{c u}^{c^{2} u} \phi(t) t^{-2} d t-\int_{u}^{c u} \phi(t) t^{-2} d t\right]$. It is not difficult to deduce from (A.1) that $[\cdots] \leqslant\left(M^{2}-m\right)\left(1-c^{-1}\right) \phi(u) / u$, and that $[\cdots]>\left(m^{2}\right.$ $-M)\left(1-c^{-1}\right) \phi(u) / u$. It follows that $D \geqslant 0$ for sufficiently small (which may be negative) $\alpha$, and that $D \leqslant 0$ for sufficiently large $\alpha$. Hence $\Phi(u) \in$ $Y[a, b]$ for some finite constants $a, b$. This proves Theorem 4 .

\section{REFERENCES}

1. R. P. Boas, Jr., Fourier series with positive coefficients, J. Math. Anal. 17 (1967), $463-483$.

2. __ Integrability theorems for trigonometric transforms, Ergebnisse der Mathematik und ihrer Grenzgebiete, Band 38, Springer-Verlag, Berlin and New York, 1967.

3. L. Y. Chan, Y. M. Chen and M. C. Liu, Some properties of asymptotic functions, Studia Math. 67 (to appear).

4. Y. M. Chen, Theorems of asymptotic approximation, Math. Ann. 140 (1960), 360-407.

5. __ On two-functional spaces, Studia Math. 24 (1964), 61-88.

6. An oscillation criterion for the second-order nonlinear differential equation $x^{\prime \prime}+$ $x F\left(x^{\prime 2}, x^{2}, t\right)=0$, Quart. J. Math. Oxford Ser. (2) 24 (1973), 165-168; Note and corrigendum, ibid. 27 (1976), 511-512.

7. P. Jain, On the integrability of power series, Proc. Amer. Math. Soc. 42 (1974), 569-574.

8. S. Koizumi, On the singular integrals. I, Proc. Japan Acad. 34 (1958), 193-198.

9. H. P. Mulholland, Concerning the generalization of the Young-Hausdorff theorem, Proc. London Math. Soc. 35 (1933), 257-293.

10. J. Németh, Generalizations of the Hardy-Littlewood inequality. II, Acta Sci. Math. 35 (1973), 127-134. 
11. L. E. Persson and I. Wik, Integrability conditions on periodic functions related to their Fourier transforms, J. Math. Anal. 44 (1973), 291-309.

12. E. Seneta, Regularly varying functions, Lecture Notes in Math., vol. 508, Springer-Verlag, Berlin and New York, 1976.

13. A. Zygmund, Trigonometric series, Vols. I and II, Cambridge Univ. Press, London and New York, 1968.

Department of Mathematics, Universtry of Hong Kong, Hong Kong 